DOI: $10.1002 /(($ please add manuscript number $))$

Article type: Communication

\title{
Photo-patterning of highly efficient state-of-the-art phosphorescent OLEDs using orthogonal hydrofluoroethers
}

Simonas Krotkus*, Fabian Ventsch, Daniel Kasemann, Alexander A. Zakhidov, Simone Hofmann, Karl Leo, and Malte C. Gather

MSc S. Krotkus, Dr. F. Ventsch, Dr. D. Kasemann, Dr. S. Hofmann, Prof. K. Leo

Institut für Angewandte Photophysik, Technische Universität Dresden, Dresden 01069, Germany

E-mail: simonas.krotkus@iapp.de

Dr. A. A. Zakhidov

Fraunhofer Research Institution for Organics, Materials and Electronic Devices COMEDD, 01109 Dresden, Germany

Prof. M. C. Gather

Institut für Angewandte Photophysik, Technische Universität Dresden, Dresden 01062 , Germany, School of Physics \& Astronomy, University of St Andrews, North Haugh, St Andrews, KY16 9SS, Scotland, UK

Keywords: orthogonal photolithography, hydrofluoroethers, phosphorescent OLEDs, doped organic layers

Together with "back plane" electronic components and highly efficient "front plane" light-emitting devices, the successful implementation of pixel patterning is a key requirement for the success of organic light-emitting diode (OLED) displays. Prerequisites for patterning techniques include cost-efficiency, scalability to large area substrates $(\geq \mathrm{Gen} 8)$, registration between multiple layers, and compatibility with state-of-the-art OLED technology. At present, state-of-the-art OLEDs are predominantly based on multilayered thin films of thermally evaporated small molecules and frequently contain phosphorescent emitters. This approach enables internal quantum efficiencies reaching unity ${ }^{[1]}$ and operational lifetimes suitable for commercial display applications. ${ }^{[2,3,4,5,6]}$ A common technique to fabricate multicolor pixelated OLED devices is shadow mask patterning. However, there are severe limitations 


\section{WILEY-VCH}

with respect to providing simple and cheap pixel alignment on large scale substrates as well as in high resolution structuring, urging a search for more suitable alternatives. ${ }^{[7]}$

In order for OLEDs to compete with liquid crystal displays (LCDs), scalable RGB pixel patterning techniques such as photolithography or ink-jet printing that are well established in LCD technology should be implemented. Various serial printing techniques including ink-jet, ${ }^{[8]}$ molecular jet ${ }^{[9]}$, and organic vapor jet printing (OVJP) ${ }^{[10]}$ were proposed for OLED displays. So far, attempts to introduce liquid printing technologies into mass production have not succeeded. OVJP is a promising alternative since it is solvent-free and compatible with multilayer phosphorescent OLEDs comprising doped organic films. However, cross-contamination of adjacent organic layers at higher pixel densities still needs to be addressed. ${ }^{[11]}$

Photolithography is a mature, cost-effective, inherently parallel patterning technique, with well-established registration protocols, high yield and resolution. Most importantly, there is a fully developed infrastructure for processing, since photolithography is the standard structuring technology in the inorganic semiconductor industry. However, up to now the application of this technique to patterning of organic semiconductor devices has remained rather limited as most of the organic materials are incompatible with the resists, etchants, and developers used in the different processing steps of conventional lithography.

One strategy to circumvent this issue is based on dry processing approaches. ${ }^{[12-13]}$ Hwang et al. ${ }^{[12]}$ used super-critical $\mathrm{CO}_{2}$ for the development of a fluorinated polymer photoresist. Electroluminescent polymer devices down to $5 \mu \mathrm{m}$ in size were demonstrated with maximum current efficiency (luminous efficacy) of $22 \mathrm{cdA}^{-1}\left(8 \mathrm{lmW}^{-1}\right)$ at $1 \mathrm{mAcm}^{-2}$. This approach, however, is limited to patterning of the hole-transporting poly(3,4ethylenedioxythiophene) poly(styrenesulfonate) (PEDOT:PSS) layers, with subsequent 


\section{WILEY-VCH}

deposition of a light-emitting polymer (LEP) layer and is thus only suitable for single color structuring. Bahlke et al. ${ }^{[13]}$ reported on lithographic patterning using frozen $\mathrm{CO}_{2}$ gas as resist. While this provides a scalable patterning approach, the use of a cooled substrate $\left(T_{\text {subs }}=77 \mathrm{~K}\right)$ during deposition of the organics leads to condensation of residual $\mathrm{H}_{2} \mathrm{O}$ and might provoke changes in thin film morphology critical for OLED performance and yield.

In some cases, traditional photoresist/solvent combinations were shown to be compatible with certain robust organic polymers. Huang et al. ${ }^{[14]}$ reported on placing commercially available photoresists SU8-10 beneath PEDOT:PSS and using a propylene glycol monomethyl ether acetate (PGMEA) developer, thus enabling pixel patterning on mechanically flexible substrates. Another approach based on conventional lithographic patterning takes advantage of the orthogonality between highly fluorinated LEPs and standard photoresists. ${ }^{[15,16,17]}$ However, the choice of materials for these traditional lithography approaches is limited, thus restricting the flexibility and performance of available OLED architectures.

Moreover, various direct-writing techniques were established which employ the organic semiconductor itself as a photoresist. Multicolor RGB pixels were demonstrated by direct structuring of solution-processed LEPs based on UV induced oxetane photocrosslinking reactions, ${ }^{[18,19,20]}$ polymer backbone cross-linking of poly( $p$-phenylene vinylene) (PPV) derivatives, ${ }^{[21]}$ as well as polymerisation of light-emitting liquid-crystalline monomers. ${ }^{[22]}$ Despite offering relatively easy implementation, reformulation of the active layer is needed in order to utilize direct photolithography. Tailoring the involved materials to be photo-patternable and at the same time exhibit high stability as well as the desired electrical and optical properties, remains very challenging. 


\section{WILEY-VCH}

Finally, protective metal layers ${ }^{[23,24]}$ or polymer films, such as Cytop ${ }^{[25]}$ or parylene $\mathrm{C}^{[26]}$ have been used to protect the organic semiconductor during conventional photolithographic processing. However, to the authors' knowledge, there are no reports to date on photolithographic patterning of OLEDs with state-of-the-art efficiency and lifetime.

In this work, we report on a patterning procedure using hydrofluoroether solvents (HFEs) and fluorinated polymers, that enables photolithographic structuring of state-of-the-art highly efficient p-i-n phosphorescent OLEDs without affecting their efficiency or lifetime. The approach is based on a modified bilayer resist concept. ${ }^{[27]}$ It relies on using a fluorinated polymer layer both as lift-off resist and as sacrificial protection layer for the organics, allowing for deposition and processing of a conventional imaging resists on top. The HFEs used for the development of the fluoropolymer are orthogonal to common solvents used for processing of organic electronic materials, and are therefore suitable for bilayer processing. In previous work, HFEs were shown to be chemically benign to most organic materials and thus enable lithographic patterning of organic semiconductors ${ }^{[28,29,27]}$ and biomolecules ${ }^{[30]}$. In addition, immersing the OLED into an HFE solution has been shown to provide effective cooling of the device, resulting in an increased efficiency and lifetime, especially at high brightness. $^{[31]}$

A simplified scheme of the patterning procedure employed in this work is presented in Figure 1a. A double layer consisting of a fluorinated polymer layer (thickness $2 \mu \mathrm{m}$ ) and an imaging resist layer (thickness $1 \mu \mathrm{m}$ ) is spin-coated onto pre-patterned indium tin oxide (ITO) coated glass substrates. The double layer is patterned by exposing defined parts of the surface to UV radiation and then developing the imaging resist. Subsequently, the pattern is transferred into the fluoropolymer layer by spin-etching in an HFE (we used a mixture of two $\mathrm{C}_{5} \mathrm{H}_{3} \mathrm{~F}_{9} \mathrm{O}$ isomers throughout this work, Figure $1 \mathrm{~b}$ ). To ensure the removal of any resist leftovers, an additional $\mathrm{O}_{2}$ plasma treatment is applied. During the transfer step, the imaging 


\section{WILEY-VCH}

resist serves as an etching mask. In the next step, the OLED stack is deposited under ultrahigh vacuum (UHV, $\sim 10^{-7} \mathrm{mbar}$ ) on top of the predefined photo-resist pattern. This procedure is followed by a lift-off step during which the entire device is immersed in HFE and the parts of the OLED stack that are on top of bilayer are removed yielding patterned organic layers.

To demonstrate the feasibility of this process, we stripe-patterned a thin film of the well-known fluorescent emitter tris(8-hydroxyquinolinato)aluminium (Alq3) (23 $\mu \mathrm{m}$ wide stripes, $25 \mu \mathrm{m}$ gap, Figure 2). As seen from the fluorescence microscopy image of the film (Figure 2a), highly regular features can be obtained. Profilometry measurements confirm that the structures exhibit well defined shapes and homogeneous thicknesses (Figure 2b). AFM scans show that the films have sharp edges, with approx. $55 \mathrm{~nm}$ of film thickness reached over less than $1.5 \mu \mathrm{m}$ in lateral dimension, in good agreement with the optical resolution of the lithography system used in this work (Figure 2c, d). Such a profile is crucial for highperformance electrical operation as deviations from a rectangular profile would lead to inhomogeneous currents running through the device. In the past it has been proven challenging to achieve sharp edges and good thickness homogeneity with other methods including $\mathrm{OVJP}^{[11]}$ and thermal patterning. ${ }^{[32]}$

Next, we investigate the impact of an HFE treatment on state-of-the art phosphorescent $\mathrm{p}$-i-n OLEDs in terms of electrical and optical characteristics as well as longterm device stability. Non-encapsulated phosphorescent red-emitting OLEDs were immersed into the HFE for $5 \mathrm{~h}$, i.e. more than twice as long as the time required for the lift-off step during patterning. Afterwards the devices were characterized and their performance was compared to the performance of untreated reference devices. The architecture of the OLED stack used for this test and for the other experiments in this work is illustrated in the inset to Figure 3a. As seen from Figure 3a, there is no detectable change in the current-voltageluminance (IVL) characteristics for the OLED immersed into HFE solution as compared to 


\section{WILEY-VCH}

the reference device. Likewise, the electroluminescence (EL) spectra are not affected by the HFE treatment (Figure 3b). We therefore conclude that the HFEs do not induce any change in the thickness of the organic layers, e.g. by swelling. Moreover, as shown in Figure 3c, the external quantum efficiency (EQE) and power efficacy (PE) of the reference and the HFE treated OLEDs are nearly identical. Only at low luminance $\left(<30 \mathrm{~cd} \mathrm{~m}^{-2}\right)$, a slight reduction in EQE and PE is observed for the HFE treated device. This corresponds to operation in the low current density regime, where slightly higher leakage currents (not visible on the logarithmic scale used in Figure 3a) are also observed for the HFE treated devices. The increase in leakage current is attributed to the additional sample handling step during which samples are immersed into HFE solvent. A more automated process is likely to further reduce the effect of HFE immersion.

In order to investigate the long-term stability of the devices after the HFE treatment, a series of OLED lifetime tests under different aging currents (i.e., different initial OLED luminance) were performed. Figure 3d summarizes the $t_{0.75}$ lifetime of the HFE treated and the reference devices for the different aging currents (the $t_{0.75}$ lifetime is the time after which the device luminance has dropped to $75 \%$ of its initial value). It can be seen that OLED lifetimes of both reference devices and pixels immersed into HFE are similar. (The slight difference in slope of the fits in Figure $3 \mathrm{~d}$ is within the range of sample-to-sample variation.) For display applications, peak luminance requirements are in the range of $100-500 \mathrm{~cd} \mathrm{~m}^{-1}$, i.e. more than one order of magnitude lower than the luminance at which the devices were operated during our test. In addition, in most display applications the pixels are not permanently operated at full brightness. The useful lifetime of OLEDs at relevant luminance levels can be estimated by assuming a power law correlation between the initial luminance $L_{0}$ and the $t_{0.75}$ lifetime. Even when assuming that the pixels are constantly operated at a luminance of $500 \mathrm{~cd} \mathrm{~m}^{-1}$, a lifetime of $t_{0.75}>100,000 \mathrm{~h}$ is expected for devices that were 


\section{WILEY-VCH}

subject to the treatment in HFE. This confirms that the used HFE is fully compatible with the state-of-the-art phosphorescent OLED stack investigated in this work.

In a next step, we show that the entire fluoropolymer based patterning process does not cause any damage to the used organic materials and thus allows fabrication of photolithographically structured devices with state-of-the-art performance. We compare OLED pixels patterned via lift-off to conventional reference OLEDs where the pixel is defined via evaporation of the materials through a shadow mask. In order to guarantee a reliable comparison of OLED characteristics, this test was performed for relatively large pixels $\left(2.5 \times 2.5 \mathrm{~mm}^{2}\right.$ reference and $2.5 \times 4.0 \mathrm{~mm}^{2}$ patterned devices $)$. The IVL characteristics (Figure 4a) as well as the EL spectra (Figure 4b) of the patterned devices are again identical (within precision of the measurement) to those of the reference pixels, suggesting that the photolithographic patterning approach based on a bilayer concept can be successfully employed to structure highly efficient OLEDs. It is worth noting that our photo-structured OLEDs achieve a peak EQE of 17\% (Figure 4c), on par with state-of-the-art phosphorescent OLEDs structured by shadow masking, and higher than the best results achieved thus far by the OVJP technique. ${ }^{[33]}$

The long-term stability of the OLEDs is not affected by the photo-patterning. Figure $4 d$ depicts the luminance decay of OLED pixels aged at a current density of $30 \mathrm{~mA} \mathrm{~cm}^{-2}$, corresponding to a high initial luminance of $6500 \mathrm{~cd} \mathrm{~m}^{-1}$. Overall, the aging behavior of photo-lithographically patterned pixels is nearly identical to the reference pixel. Only a slight initial decrease in luminance can be observed for the photo-lithographically patterned device over the first few hours. Nevertheless, stable long-term operation of patterned OLEDs is demonstrated for over $1000 \mathrm{~h}$. 


\section{WILEY-VCH}

Finally, we demonstrate the capability of our photolithographic approach to structure electroluminescent organic devices on a scale relevant for OLED displays (Figure 5). In display applications, pixel sizes in the range of 30-100 $\mu \mathrm{m}$ are used which requires defining stripes of the organic material with a width in the same range. Here, we show structuring and alignment of OLED stripes with a width of $65 \mu \mathrm{m}$ placed on top of $15 \mu \mathrm{m}$ wide pre-patterned ITO anode stripes (Figure 5a). Well-defined stripes with homogeneous thickness are obtained as indicated by the excellent brightness homogeneity in the fluorescence micrograph

(Figure 5b). Under electrical operation, homogenous EL is observed from the area of the film located on top of the ITO contact (Figure 5c). The electroluminescence spectrum emitted by the patterned device is comparable to the spectrum obtained from pixels evaporated though a shadow mask (Figure 5d).

In conclusion, we have demonstrated photolithographic patterning of a state-of-the-art multilayered phosphorescent OLED structure comprising doped charge transport layers.

Photolithographic patterning was enabled by the use of a fluoropolymer film for lifting off the active layers in an HFE solvent, in combination with conventional processing of a photoresist/developer to allow pattern transfer. Electroluminescent structures down to $20 \mu \mathrm{m}$ in size were demonstrated, exceeding the resolution requirements for most display applications. In contrast to many of the previously reported lithographic approaches to the patterning of organic devices, no measurable detrimental effect on device performance and long term stability was found after patterning the whole OLED stack via lift-off in HFE. Our results indicate that treatment in environmentally friendly HFE solvents is fully compatible with thermally evaporated p-i-n OLED technology and enables photolithographic patterning, thus holding great promise for high resolution and large area multicolor structuring of highly efficient OLED displays. 


\section{WILEY-VCH}

\section{Experimental Section}

Photolithographic patterning protocol. At first, a layer of highly fluorinated polymer (Ortho 310 , Orthogonal Inc.) is spin-coated $(800 \mathrm{rpm}, 60 \mathrm{~s})$ under ambient conditions on a glass/ITO substrate. Next, a layer of a commercially available imaging resist $(A Z \circledast n L O F-2020$, negative tone resist, AZ Electronic Materials) is spin-coated (3000 rpm, $30 \mathrm{~s}$ ) on top. Mutual orthogonality between these two layers ensures that processing of the imaging resist does not alter the properties of the fluorinated polymer and vice versa. Patterns are transferred to the imaging resist by exposing the samples with UV radiation using an SF-100 UV broadband exposure system. Subsequent development in TMAH based developer and rinsing in distilled water removes exposed parts of the imaging resist. The pattern transfer to the fluoropolymer layer is done via spin-etching with HFE (Novec 7100, 3M). To ensure a complete removal of the fluorinated polymer, an additional $\mathrm{O}_{2}$ plasma etching step is carried out for $10 \mathrm{~min}$. Finally, OLEDs are deposited on top of the pre-patterned bilayer resist as described below. The areas of the OLED stack with fluoropolymer underneath are subsequently removed via lift-off in HFE. We find that approximately $2 \mathrm{~h}$ treatment in HFEs is necessary to fully remove the fluoropolymer layer.

OLED fabrication. Organic materials were commercially purchased and purified by vacuum gradient sublimation. Prior to device fabrication, the pre-structured ITO (thickness, $90 \mathrm{~nm}$ ) coated glass substrates are cleaned using ultrasonic treatment in N-Methyl-2-pyrrolidon, distilled water, and ethanol. Organic and metal layers are deposited by thermal evaporation in a UHV chamber (Kurt J. Lesker Co.) at a base pressure of $10^{-7}$ mbar without breaking the vacuum. Evaporation rates and thicknesses of all layers are measured in situ via quartz crystals. Doping is achieved by co-evaporation. The OLED stack used in this work consists of a hole transport layer (60 nm) of 2,2',7,7'-Tetrakis-(N,N-di-methylphenylamino)-9,9'spirobifluoren (Spiro-TTB) doped with 4 \%wt 2,2'-(perfluoronaphthalene-2,6- 


\section{WILEY-VCH}

diylidene)dimalononitrile (F6-TCNNQ), an electron blocking layer (10 nm) of N,N'Di(naphthalen-1-yl)-N,N'-diphenyl-benzidine ( $\alpha$-NPD), an emission layer $(20 \mathrm{~nm})$ composed of $\alpha$-NPD doped with $10 \mathrm{wt} \%$ of the red phosphorescent emitter iridium(III)bis(2methyldibenzo-[f,h]chinoxalin)(acetylacetonat) (Ir(MDQ) 2 (acac)), a hole blocking layer $(10 \mathrm{~nm})$ of aluminum(III)bis(2-methyl-8-quninolinato)-4-phenylphenolate (BAlq 2 ) and an electron transport layer of 4,7-diphenyl-1,10-phenanthroline (BPhen) doped with cesium (Cs). The OLED is completed by depositing a $100 \mathrm{~nm}$ thick aluminum (Al) cathode. After processing and lift-off (see above), the OLEDs are encapsulated in nitrogen atmosphere using glass lids and UV-curing epoxy resin. The active area of the reference and the patterned devices is $6.49 \mathrm{~mm}^{2}$ and $10 \mathrm{~mm}^{2}$, respectively.

Characterization of the devices. All measurements of the encapsulated OLED are carried out under ambient conditions. Current-voltage-luminance characteristics are measured using a source measure unit (Keithley SMU 2400) and a calibrated Si-photodiode. The spectral radiance is recorded with a calibrated spectrometer (Instrument Systems GmbH CAS140). Efficiencies are calculated using Lambertian assumption. Luminance decay curves are acquired by aging OLEDs under constant current condition The OLED lifetime at low initial luminance is extrapolated using (Equation 1):

$$
L_{0}^{n} \cdot t_{0.75}=\text { const }
$$

where $n \approx 2.2$ is the empirically found acceleration factor. The electroluminescence micrograph depicted in Figure 5c is taken using an optical microscope (Carl-Zeiss-Jena Jenaval). Photoluminescence micrographs and topography profiles are recorded using a fluorescence microscope and a Veeco Dektak 150 profilometer, respectively. AFM scans are acquired with CombiScope 1000 (AIST-NT) measurement setup.

\section{Acknowledgements}

The authors kindly acknowledge the financial support by the Free State of Saxony, the Sächsische Aufbaubank (SAB, Project Nr. 100087862), and the European Social Fund (ESF). 


\section{WILEY-VCH}

Received: ((will be filled in by the editorial staff))

Revised: ((will be filled in by the editorial staff)) Published online: ((will be filled in by the editorial staff))

[1] C. Adachi, M. A. Baldo, M. E. Thompson, S. R. Forrest, J. Appl. Phys. 2001, 90, 5048.

[2] R. Meerheim, S. Scholz, S. Olthof, G. Schwartz, S. Reineke, K. Walzer, K. Leo, J. Appl. Phys. 2008, 104, 014510.

[3] I. R. de Moraes, S. Scholz, B. Lüssem, K. Leo, Org. Electron. 2012, 13, 1900.

[4] M. A. Baldo, S. Lamansky, P. E. Burrows, M. E. Thompson, S. R. Forrest, Appl. Phys. Lett. 1999, 75, 4.

[5] X. Zhou, D. S. Qin, M. Pfeiffer, J. Blochwitz-Nimoth, A. Werner, J. Drechsel, B. Maennig, K. Leo, M. Bold, P. Erk, H. Hartmann, Appl. Phys. Lett. 2002, 81, 4070.

[6] G. He, O. Schneider, D. Qin, X. Zhou, M. Pfeiffer, K. Leo, J. Appl. Phys. 2004, 95, 5773.

[7] J. H. Kwon, Inf. Disp. 2013, 29, 12.

[8] H. Sirringhaus, T. Kawase, R. H. Friend, T. Shimoda, M. Inbasekaran, W. Wu, E. P. Woo, Science 2000, 290, 2123.

[9] J. Chen, V. Leblanc, S. H. Kang, P. J. Benning, D. Schut, M. A. Baldo, M. A. Schmidt, V. Bulović, Adv. Funct. Mater. 2007, 17, 2722.

[10] M. Shtein, P. Peumans, J. B. Benziger, S. R. Forrest, Adv. Mater. 2004, 16, 1615.

[11] G. J. McGraw, S. R. Forrest, Adv. Mater. 2013, 25, 1583.

[12] H. S. Hwang, A. A. Zakhidov, J.-K. Lee, X. André, J. A. DeFranco, H. H. Fong, A. B. Holmes, G. G. Malliaras, C. K. Ober, J. Mater. Chem. 2008, 18, 3087.

[13] M. E. Bahlke, H. A. Mendoza, D. T. Ashall, A. S. Yin, M. A. Baldo, Adv. Mater. 2012, 24, 6136.

[14] J. Huang, R. Xia, Y. Kim, X. Wang, J. Dane, O. Hofmann, A. Mosley, A. J. de Mello, J. C. de Mello, D. D. C. Bradley, J. Mater. Chem. 2007, 17, 1043.

[15] J.-K. Lee, H. H. Fong, A. A. Zakhidov, G. E. McCluskey, P. G. Taylor, M. SantiagoBerrios, H. D. Abruña, A. B. Holmes, G. G. Malliaras, C. K. Ober, Macromolecules 2010, 43, 1195. 


\section{WILEY-VCH}

[16] H. H. Fong, J.-K. Lee, Y.-F. Lim, A. A. Zakhidov, W. W. H. Wong, A. B. Holmes, C. K. Ober, G. G. Malliaras, Adv. Mater. 2011, 23, 735.

[17] A. A. Zakhidov, J.-K. Lee, J. A. DeFranco, H. H. Fong, P. G. Taylor, M. Chatzichristidi, C. K. Ober, G. G. Malliaras, Chem. Sci. 2011, 2, 1178.

[18] C. D. Müller, A. Falcou, N. Reckefuss, M. Rojahn, V. Wiederhirn, P. Rudati, H. Frohne, O. Nuyken, H. Becker, K. Meerholz, Nature 2003, 421, 829.

[19] M. C. Gather, A. Köhnen, A. Falcou, H. Becker, K. Meerholz, Adv. Funct. Mater. 2007, $17,191$.

[20] M. C. Gather, N. M. Kronenberg, K. Meerholz, Adv. Mater. 2010, 22, 4634.

[21] X. Deng, K. Y. Wong, Macromol. Rapid Commun. 2009, 30, 1570.

[22] M. P. Aldred, A. E. A. Contoret, S. R. Farrar, S. M. Kelly, D. Mathieson, M. O’Neill, W. C. Tsoi, P. Vlachos, Adv. Mater. 2005, 17, 1368.

[23] P. F. Tian, P. E. Burrows, S. R. Forrest, Appl. Phys. Lett. 1997, 71, 3197.

[24] B. Lamprecht, E. Kraker, G. Weirum, H. Ditlbacher, G. Jakopic, G. Leising, J. R. Krenn, Phys. Status Solidi RRL - Rapid Res. Lett. 2008, 2, 16.

[25] J.-F. Chang, M. C. Gwinner, M. Caironi, T. Sakanoue, H. Sirringhaus, Adv. Funct. Mater. 2010, 20, 2825.

[26] J. A. DeFranco, B. S. Schmidt, M. Lipson, G. G. Malliaras, Org. Electron. 2006, 7, 22.

[27] H. Kleemann, A. A. Zakhidov, M. Anderson, T. Menke, K. Leo, B. Lüssem, Org. Electron. 2012, 13, 506.

[28] A. A. Zakhidov, J.-K. Lee, H. H. Fong, J. A. DeFranco, M. Chatzichristidi, P. G. Taylor, C. K. Ober, G. G. Malliaras, Adv. Mater. 2008, 20, 3481.

[29] P. G. Taylor, J.-K. Lee, A. A. Zakhidov, M. Chatzichristidi, H. H. Fong, J. A. DeFranco, G. G. Malliaras, C. K. Ober, Adv. Mater. 2009, 21, 2314.

[30] K. M. Midthun, P. G. Taylor, C. Newby, M. Chatzichristidi, P. S. Petrou, J.-K. Lee, S. E. Kakabakos, B. A. Baird, C. K. Ober, Biomacromolecules 2013, 14, 993.

[31] A. A. Zakhidov, S. Reineke, B. Lüssem, K. Leo, Org. Electron. 2012, 13, 356.

[32] D. Goto, M. Mohri, K. Morimoto, A. Mzhavia, C. Adachi, Adv. Opt. Mater. 2013, doi: 10.1002/adom.201300372

[33] G. J. McGraw, D. L. Peters, S. R. Forrest, Appl. Phys. Lett. 2011, 98, 013302. 


\section{WILEY-VCH}

(a)

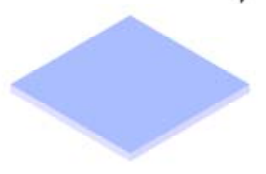

1)
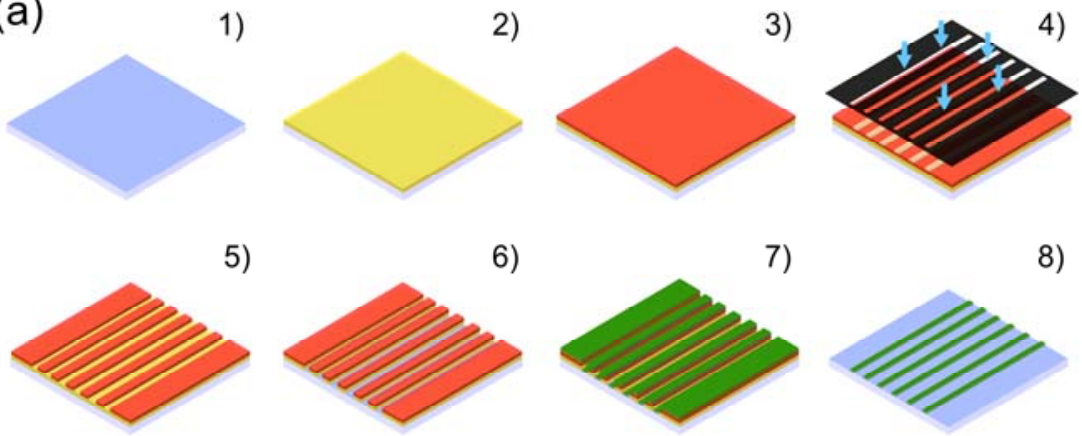

6)

5)

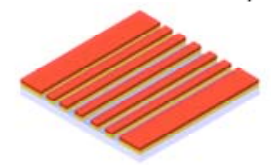

7)

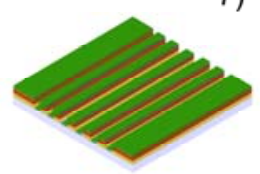

(b)

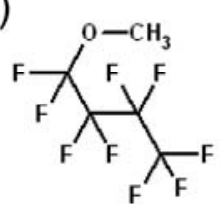

8)

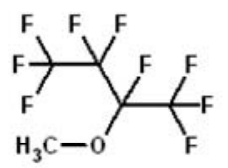

Figure 1. (a) Schematic illustration of the photolithographic patterning process using a fluoropolymer and a hydrofluoroether solvent (HFE) for development and lift-off: 1) substrate, 2) spin-coating of fluoropolymer layer, 3) spin-coating of imaging resist, 4) UV exposure through mask, 5) development of imaging resist, 6) pattern transfer to fluoropolymer in HFE, 7) OLED deposition, 8) lift-off in HFE; (b) chemical structure of the HFE used in this study comprising of a mixture of two $\mathrm{C}_{5} \mathrm{H}_{3} \mathrm{~F}_{9} \mathrm{O}$ isomers.

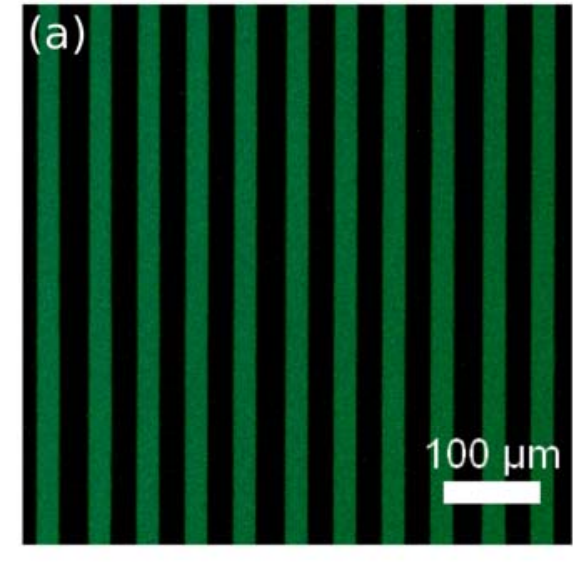

(c)

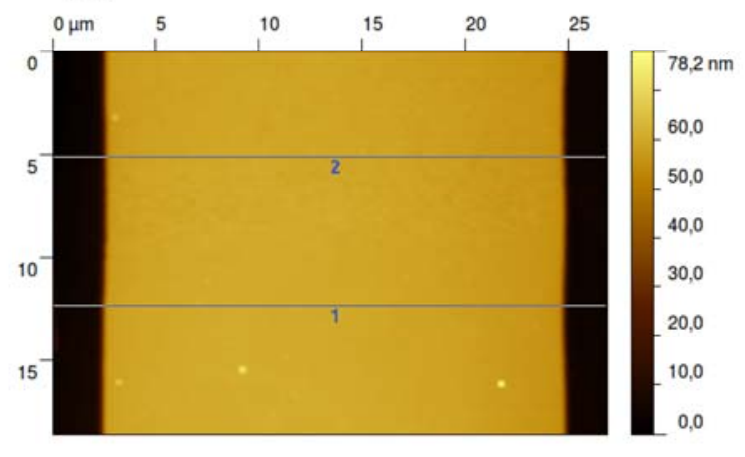

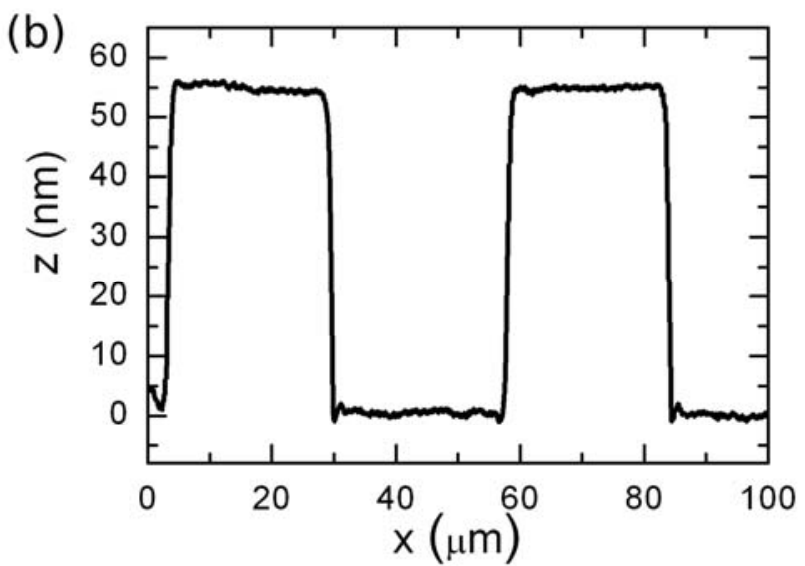

(d)

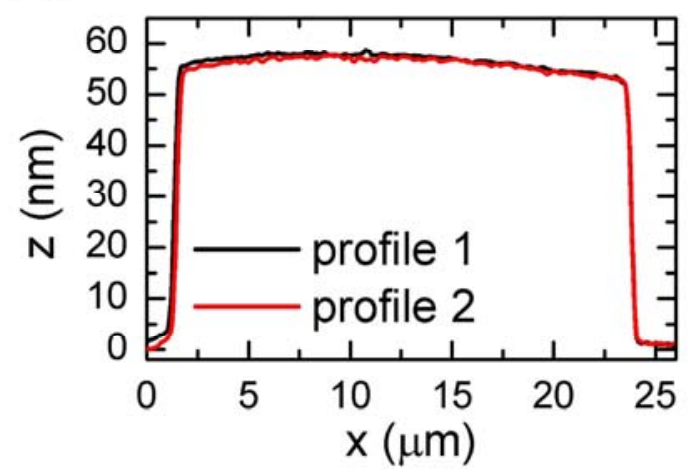

Figure 2. (a) Photoluminescence micrograph of a patterned Alq3 thin film and (b) corresponding cross-section topography profile. (c) AFM scan of the structured feature and (d) corresponding edge profiles. 


\section{WILEY-VCH}

(a)

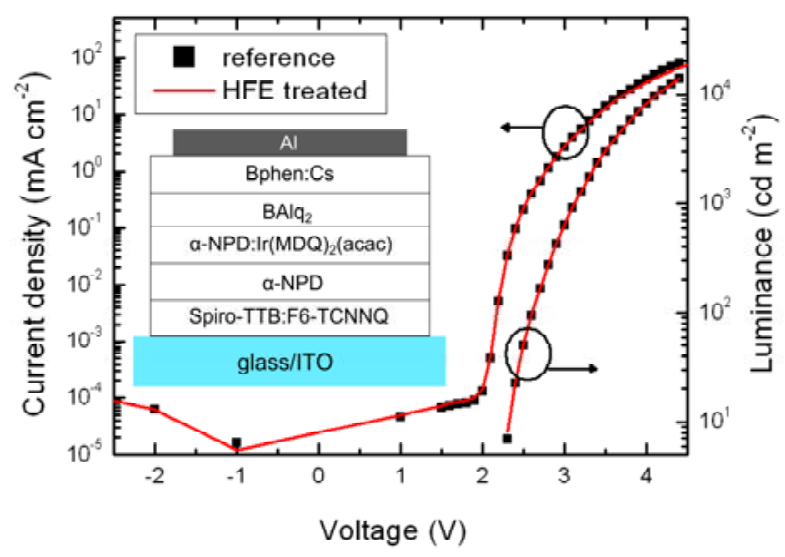

(c)

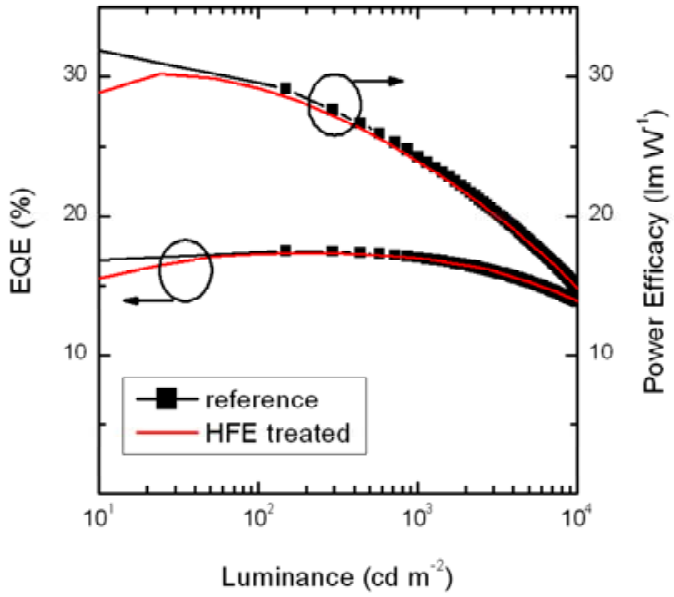

(b)

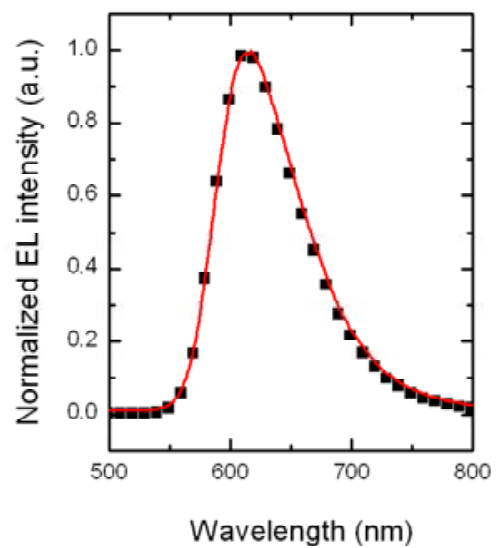

(d)

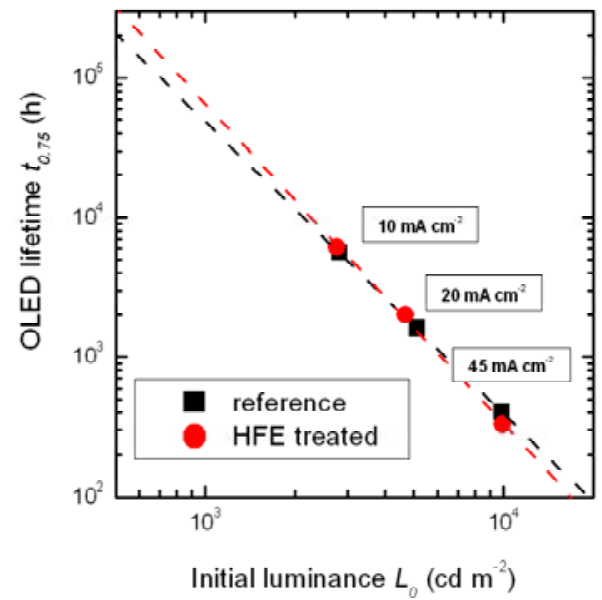

Figure 3. (a) Current density-voltage-luminance characteristics of OLEDs treated for $5 \mathrm{~h}$ in HFE (solid red line) and non-treated reference device (black squares); a schematic of the OLED stack used in this work is shown in the insert; (b) electroluminescence spectra, (c) external quantum efficiency and power efficacy vs. luminance of the respective devices. (d) The $t_{0.75}$ lifetime vs. initial luminance $L_{0}$ for treated and untreated devices. 


\section{WILEY-VCH}

(a)

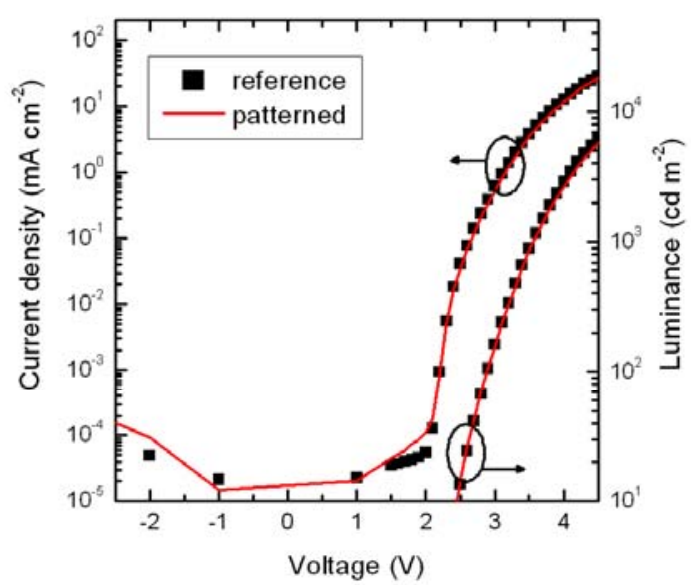

(c)

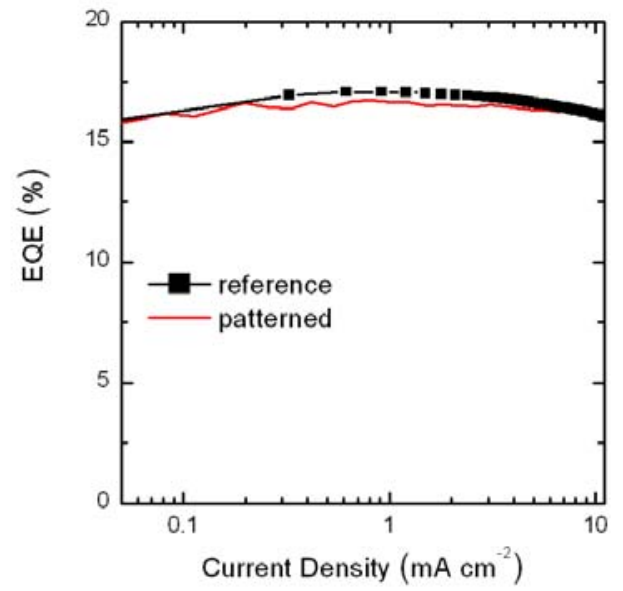

(b)

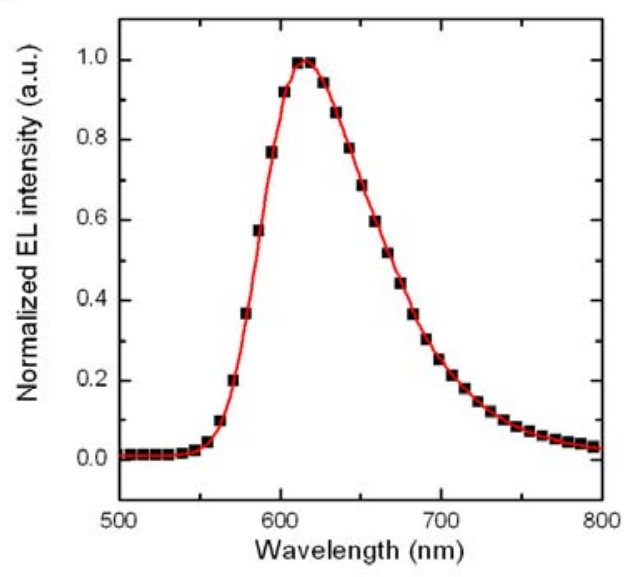

(d)

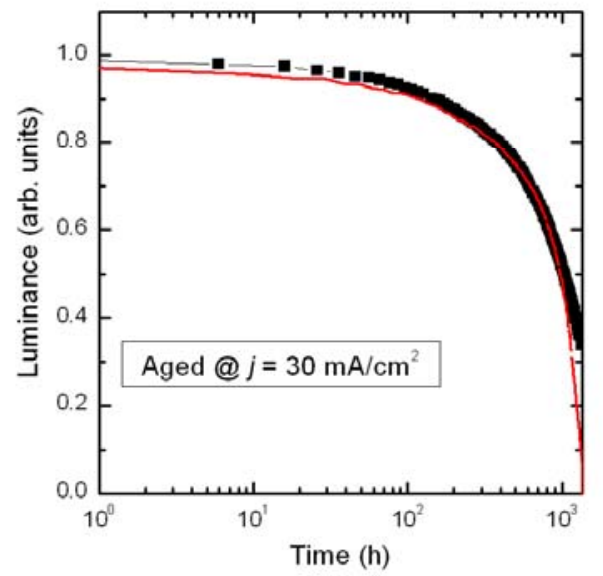

Figure 4. (a) Current density-voltage-luminance characteristics and (b) electroluminescence spectra of lithographically patterned pixels using HFE (solid red line) and reference pixels patterned by evaporation through a shadow mask (black squares). (c) External quantum efficiency (EQE) vs. current-density of the same devices. (d) Normalized luminance decay of patterned OLED pixel and reference, aged at a current density of $30 \mathrm{~mA} / \mathrm{cm}^{2}$. 


\section{WILEY-VCH}

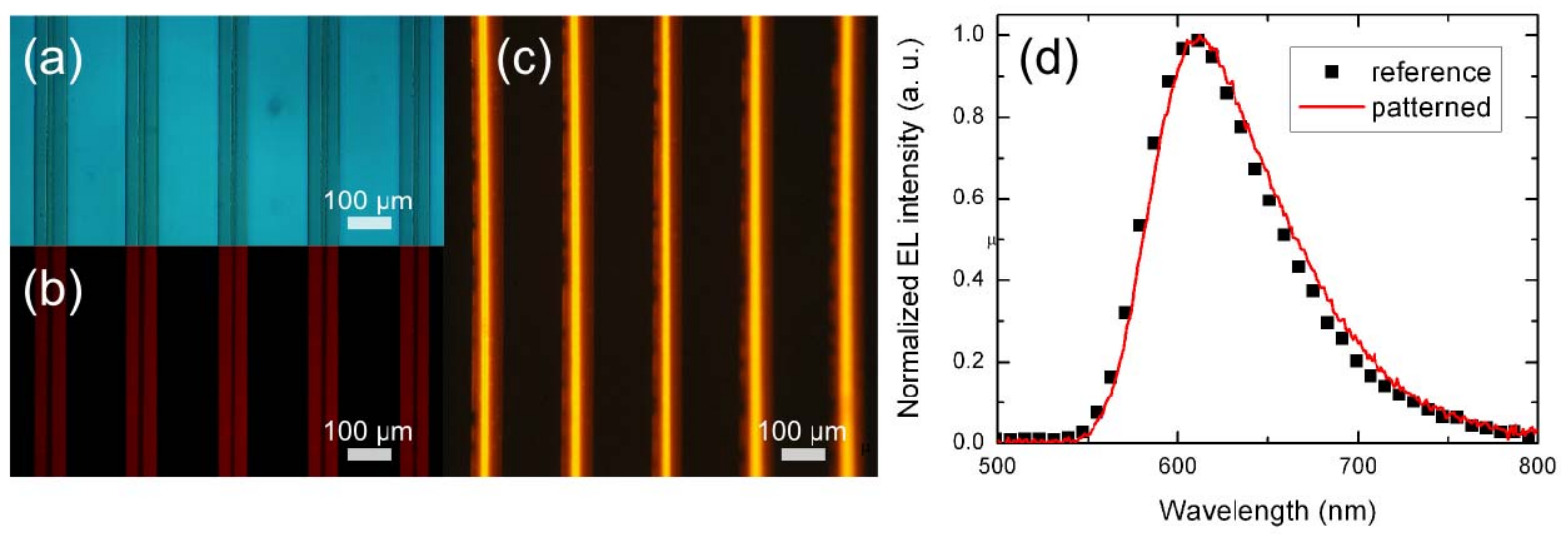

Figure 5. (a) Bright field and (b) fluorescence micrograph of $65 \mu \mathrm{m}$ wide micro-patterned stripes of red-phosphorescent OLEDs on a glass substrate with $15 \mu \mathrm{m}$ wide ITO stripes. (c) Micrograph of photo-lithographically patterned OLEDs under electrical operation. (d) Electroluminescence spectra of micro-patterned device (red solid line) and unpatterned reference OLED (black squares). 
Photolithography offers a scalable solution for large area pixel patterning of organic light-emitting diode (OLED) displays. However, so far its use has been limited due to the incompatibility of traditional processing steps with the majority of organic materials. In this work, photolithographic structuring of state-of-the-art phosphorescent red p-i-n OLEDs is demonstrated using fluorinated polymers in combination with hydrofluoroether solvents (HFEs). This is the first report on photolithographically patterned OLEDs with state-of-the-art efficiency $\left(\mathrm{EQE}_{\max } \sim 17 \%\right)$ and good long-term stability $\left(t_{0.75}>100000 \mathrm{~h}\right.$ at $\left.500 \mathrm{~cd} \mathrm{~m}^{-2}\right)$.

\section{Keyword}

Orthogonal photolithography, hydrofluoroethers, phosphorescent OLEDs, doped organic layers

S. Krotkus*, F. Ventsch, D. Kasemann, A. A. Zakhidov, S. Hofmann, K. Leo and M. C. Gather

Photolithographic patterning of highly efficient state-of-the-art phosphorescent OLEDs

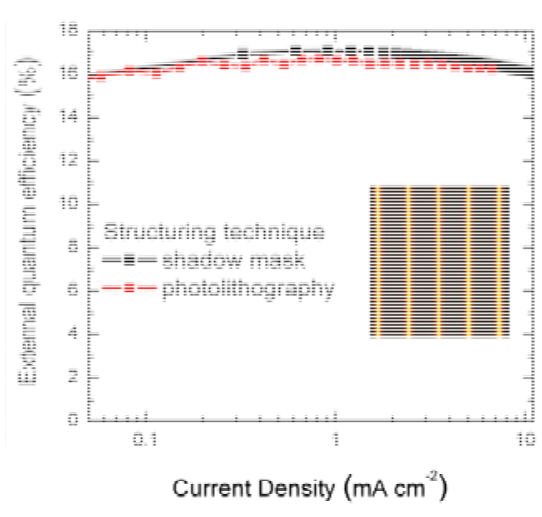

